\title{
Primary Cutaneous Diffuse Large B-Cell Lymphoma-Leg Type, a Convoluted Path to Diagnosis
}

\author{
McSwain KM*, Meade J D and Prakash S \\ Florida State University College of Medicine, USA \\ *Correspondling author: Kiah McSwain, Florida State \\ University College of Medicine, Candidate Class of 2022, \\ 7431 Roebelenii Ct. Sarasota, FL 34241, USA
}

Received: December 27, 2020; Accepted: February 04, 2021; Published: February 11, 2021

\begin{abstract}
Primary cutaneous diffuse large B cell lymphoma is a rare and aggressive form of primary cutaneous B-cell lymphoma. It typically presents with reddish to brown-nodules on the distal legs. We introduce the case of a 71 year-old female who presented to an urgent care clinic with a new onset of painful nodules on her right lower extremity. After workup and treatment for superficial thrombophlebitis, the patient improved until she presented to her primary care physician with worsening symptoms one month later. Diagnosis of primary cutaneous diffuse large B-cell lymphoma, leg type was confirmed via biopsy. After referral to oncology, further workup showed stage 4 disease. The patient was subsequently scheduled to begin treatment with R-CHOP. Unfortunately, prior to the initiation of chemotherapy she passed away after contracting COVID-19 pneumonia.
\end{abstract}

\section{Background}

Primary Cutaneous Diffuse Large B Cell Lymphoma-Leg Type (PCDLBCL-LT) accounts for approximately $5-10 \%$ of all diffuse large $\mathrm{B}$ cell lymphomas and are known to more commonly occur in females [1]. Cutaneous lymphomas can be described as being either primary or secondary [2]. Primary cutaneous B cell lymphomas have a much more aggressive course, and often do not display any symptoms such as pain, burning or itching upon presentation. The diagnosis may initially be challenging to make, as the presentation often mimics other more common etiologies such as superficial thrombophlebitis and cellulitis. The median age of diagnosis is about 78 years of age [3]. Diagnosis can be confirmed with the appropriate clinical picture and consistent immunohistological findings [4-6]. PCDLBCL-LT is characterized by invasion of immunoblastic cells in the dermis with little to no epidermotropism [3]. PCDLBCL-LT commonly expresses BCL-2 antigenic proteins, along with MUM-1 and FOXP-1. Certain studies have associated the expression of certain antigenic proteins and proto-oncogenes with survival outcomes in patients diagnosed with PCDLBCL-LT. A recent study by Hallerman et al., found that patients who had a positive IHC stain for BCL-2 and MUM-1 had a poorer prognosis than patients who stained positive for BCL-6.

\section{Case Presentation}

A 71-year-old female presented to her primary care provider for a follow-up visit 2-days after presenting to a walk-in clinic with a new onset of large, painful, skin-colored lesions on her lower extremities (Figure 1). The patient had a past medical history significant for hypertension, arthritis, and cervical diffuse large B-cell lymphoma (activated type), diagnosed in 2017, for which she underwent 6 cycles of R-CHOP therapy with complete response seenon PET in 2018. Upon her visit to the clinic, the patient had noticed tender nodules on her right lower extremity that had been getting progressively worse over the past few weeks. She was evaluated and ruled out for deep vein thrombosis in the urgent care clinic setting initially. At presentation, Lesions appeared skin-colored, swollen, bumpy, and

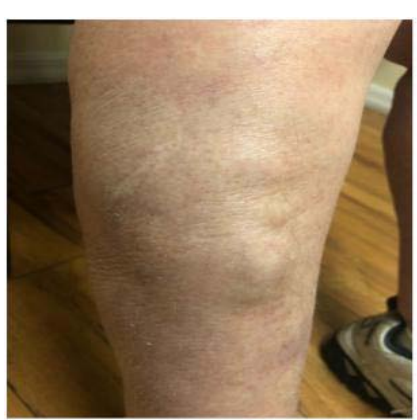

Figure 1:

warm. The patient endorsed symptoms of mild muscle weakness and fatigue, but she denied any systemic symptoms of fever, chills, night sweats, or weight loss. The patient also denied any trauma to the area. Ultrasound of the lower extremity showed extensive subcutaneous soft tissue swelling, read to be consistent with cellulitis. She was treated with compression stockings and $500 \mathrm{mg}$ of cephalexin in anticipation of a diagnosis of superficial thrombophlebitis. At followup, symptoms had improved. However, two weeks later the patient presented again with worsening leg pain, associated with larger and now violaceous right lower extremity lesions with new left lower extremity discoloration. She was subsequently referred to vascular surgery and infectious disease for further workup. After ruling out infectious and vascular etiologies she underwent bilateral skin biopsies, revealing primary cutaneous diffuse large B-cell lymphoma, leg type. Immunostaining was positive for CD20, PAX-5, BCL-2, BCL-6, and MUM-1. Resultant analysis demonstrated MYC 8q24 rearrangement in $88 \%$ of the cell analyzed. After diagnosis, she was referred to oncology who further staged her at Stage 4 primary skin lymphoma and ruled out this diagnosis as a recurrence of previous disease. Per oncology, she was scheduled to begin R-CHOP therapy once more. Prior to the initiation of chemotherapy, follow-up CT scan of chest, abdomen, and pelvis revealed an incidental finding of bilateral basilar infiltrates with associated scattered ground glass
J Fam Med - Volume 8 Issue 1 - 2021

ISSN : 2380-0658 | www.austinpublishing group.com

McSwain et al. (C) All rights are reserved
Citation: McSwain KM, Meade JD and Prakash S. Primary Cutaneous Diffuse Large B-Cell Lymphoma-Leg Type, a Convoluted Path to Diagnosis. J Fam Med. 2021; 8(1): 1238. 
opacities suggestive of an atypical or viral pneumonia. Although asymptomatic, this finding prompted COVID-19 testing. The patient later tested positive and experienced complications of COVID-19 pneumonia and passed 9 days after her incidental CT findings.

\section{Discussion}

PCDLBCL-LT is a rare form of lymphoma classified under the umbrella of primary cutaneous B-cell lymphomas. Like their visceral counterparts there are several variants to the primary cutaneous B-cell lymphomas with the most common being Primary Cutaneous Follicular Center Lymphoma (PCFCL) and primary cutaneous marginal zone lymphoma (PCMZL) [7]. Each primary cutaneous lymphoma presents very similarly with an eruption of violaceous papules or plaques. However, discrete reddish-brown nodules on the lower limbs is a presentation that is shared among PCFCL and PCDLBCL-LT. Distinguishing between subtypes is important in determining prognosis and treatment. According to the 2018 World Health Organization's European Organization of Research and Treatment of Cancer (EORTC) classification, PCFCL makes up 12\% of primary cutaneous lymphomas. PCMZL is less common with a relative frequency of $9 \%$, and PCDLBCLT-LT is among the rarest primary cutaneous B-cell lymphomas with a frequency at around 4\%. Furthermore, PCDLBCLT-LT has the lowest approximate 5-year survival rate at $56 \%$ while PCMZL and PCFCL have a much better prognosis with 5 -year survival rates of $95 \%^{+}$or greater. Treatment varies among these lymphomas as well. PCFCL has been shown to respond well to local excision and radiotherapy [8,9]. This is in contrast to PCDLBCL-LT, which requires a more extensive chemotherapy regimen consisting of rituximab, cyclophosphamide, prednisone, doxorubicin, and vincristine (R-CHOP).

We present a case of a complicated path to diagnosis of primary cutaneous diffuse large B-cell lymphoma, leg type. Various factors lead to the delay of care, including a presentation mimicking superficial thrombophlebitis, diagnostic studies suggesting cellulitis, and improvement with preliminary anti-infective and inflammatory agents. Ultimately, this case culminated in the patient's untimely death shortly after incidental CT findings were consistent with COVID-19 pneumonia. Uniquely, this patient had a history of nodal lymphoma, successfully diagnosed and treated in 2018. Through histological and oncologic workup, this patient was determined to have a new primary cutaneous lymphoma, separate from her previous disease. There was no evidence of a systemic, metastatic, or nodal disease that secondarily involved the skin.

\section{Conclusion}

PCDLBCL-LT is a particularly aggressive form of lymphoma, especially if associated with BCL-2 positivity. Clinicians may be at a disadvantage when initially confronted with this disease process, as it may mimic many other more common etiologies. With a lack of clinical data, and no specific clinical criteria to diagnose this rare disease, a high index of suspicion is needed. Our hope is that clinicians can keep this on their differential when confronted with a similar patient presentation.

\section{References}

1. Gopal MM, Malik A. Primary cutaneous diffuse large B-cell lymphoma of the upper limb: a fascinating entity. Indian J Dermatol. 2013; 58: 366-368.

2. Willemze R, Jaffe ES, Burg G, Cerroni L, Berti E, Swerdlow SH, et al. WHOEORTC classification for cutaneous lymphomas. Blood. 2005; 105: 3768 3785.

3. Dominis M, Dzebro S, Gasparov S, Pesut A, Kusec R. Diffuse large B-cel lymphoma and its variants. Croat Med J. 2002; 43: 535-540.

4. Kerl H, Fink-Puches R, Cerroni L. Diagnostic criteria of primary cutaneous B-cell lymphomas and pseudolymphomas. Keio J Med. 2001; 50: 269-273.

5. Hallermann C, Niermann C, Fischer RJ, Schulze HJ. New prognostic relevant factors in primary cutaneous diffuse large B-cell lymphomas. J Am Acad Dermatol. 2007; 56: 588-597.

6. Wobser M, Kneitz H, Brocker EB, Becker JC. Primary cutaneous diffuse large B-cell lymphoma, leg-type, treated with a modified R-CHOP immunochemotherapy - diagnostic and therapeutic challenges. J Dtsch Dermatol Ges. 2011; 9: 204-211.

7. Patsatsi A, Kyriakou A, Karavasilis V, Panteliadou K, Sotiriadis D. Primary cutaneous diffuse large B-cell lymphoma, leg type, with multiple local relapses: case presentation and brief review of literature. Hippokratia. 2013; 17: 174-176.

8. Senff NJ, Noordijk EM, Kim YH, Bagot M, Berti E, Cerroni L, et al. European Organization for Research and Treatment of Cancer; International Society for Cutaneous Lymphoma. European Organization for Research and Treatment of Cancer and International Society for Cutaneous Lymphoma consensus recommendations for the management of cutaneous B-cell lymphomas. Blood. 2008; 112: 1600-1609.

9. Willemze R, Cerroni L, Kempf W, Berti E, Facchetti F, Steven H, et al The 2018 update of the WHO-EORTC classification for primary cutaneous lymphomas. Blood. 2019; 133: 1703-1714. 